\title{
Editorial Especial
}

\section{Uma antropologia da interface: políticas públicas e assistenciais em perspectiva comparativa \\ An anthropology of interface: a comparative perspective on public and care policies}

\section{Chiara Pussetti}

Pesquisadora Associada do Instituto de Ciências Sociais da Universidade de Lisboa e Professora Visitante da Universidade Federal do Rio Grande do Norte.

Embora uma parte substantiva da produção nas ciências sociais tenha se concentrado precisamente nas resistências e dificuldades da integração dos elementos da população considerados como marginais e/ou vulneráveis em estruturas sociais preexistentes (processos de discriminação, controle, punição e exclusão), já o complexo dispositivo assistencialista que se construiu em torno desse público é frequentemente alvo de menor interesse. No entanto, nas últimas décadas, disponibilizaram-se importantes estruturas de financiamento e mobilizaram-se os mais diversos agentes e campos de expertise no sentido de criar e expandir uma rede de cuidados sociais, notadamente de saúde, ou em nome do direito à saúde, responsável pela intervenção junto de populações desfavorecidas e excluídas.

O nosso projeto comum - financiado pela Capes (Coordenadoria de Aperfeiçoamento do Pessoal de Nível Superior) no Brasil ${ }^{1}$ e pela FCT (Fundação para à Ciência e Tecnologia ) em Portugal ${ }^{2}$, com coordenação do professor Rubens de C. F. Adorno, responsável do grupo de pesquisa ligado à Universidade de São Paulo (USP) e por mim, pelo que diz respeito à equipa do Centro em Rede de Investigação em Antropologia (CRIA/ISCTE/IUL) - foi dedicado justamente à análise das intervenções sociais que gravitam em torno do binómio vulnerabilidade/risco, que por sua vez se ex- pressa numa outra dicotomia assistência/segurança.

A arquitetura conceitual do projeto dialoga criticamente com a línea de análise inspirada pelo tema foucaultiano de "governamentalidade" que inspirou, nas últimas duas décadas, trabalhos relevantes sobre: espaço e habitação (Rabinow, 1989); psiquiatria, medicina e construção do self (Ong, 1995; Rose, 1998); pobreza e insegurança (Dean, 1991; Procacci, 1993); insegurança social (Ewald, 1986); risco (O'Malley, 1992; Lupton, 2005); regulação da reprodução, controle do corpo e da sexualidade (Horn, 1994; Weir, 1996; Ruhl, 1999; Greenhalgh, 2003); imigração e segurança (Bigo, 2011; Duffield, 2007; Huysmans e col., 2006); saúde global (King, 2002; Roberts, 2010; Sargent e Larchanché, 2011) e religião (Garmany, 2010; Biebricher, 2011; Eves, 2011).

Se muitos estudos na área das ciências sociais focaram a questão da integração das populações consideradas como marginais, vulneráveis e/ou de risco, trabalhando temas ligados à securitização das fronteiras, à criminalização das periferias ou dos imigrantes não documentados (Anderson, 2000; Duffield, 2001; Coutin, 2003; Silverstein, 2005; Graham, 2002; Bigo e Guild, 2005; Fischer, 2010; Khosravi, 2010; Fassin, 2011; Inda, 2011), o complexo dispositivo de cuidados dirigido às populações consideradas como desfavorecidas - nos seus tons ambíguo de proteção e correção, empoderamento e contenção - recebeu substancialmente menos interesse. Se é claro que essa preocupação revela a sobreposição de interesses sociais e de saúde pública com questões de segurança e vigilância de populações potencialmente de risco, o discurso contemporâneo da compaixão e da intervenção - o que definimos em outros trabalhos como economia moral da benevolência (Pussetti e Brazzabeni, 2011; Pussetti e Barros, 2012) - é possivelmente o exemplo mais evidente de controle liberal-cosmopolita e de construção da responsabilidades sociais e individuais dos nossos tempos. 
Os trabalhos desenvolvidos no projeto mostram claramente como a pobreza ou a marginalidade social estão sendo reformuladas e transformadas de um problema de desigualdade socioeconômica de classe e de má distribuição de riqueza para uma questão profundamente racializada de desvio e debilidade moral, de patologia cultural e de dependência crónica.

As diferentes pesquisas desenvolvidas numa ótica comparativa entre Brasil e Portugal evidenciam que, por um lado, existe uma representação que habitualmente relaciona as populações carenciadas e/ou excluídas com desvio, falta de competências sociais e valores morais, criminalidade e insegurança, constituindo desse modo um risco para a sociedade. Por outro lado, essas populações são também consideradas como grupos em dificuldade, necessitados, dependentes e desprotegidos, interpretados como se vivendo em um estado permanente de vulnerabilidade. A assunção pedagógica implícita nessa representação é que essas pessoas devem ser educadas para se tornar sujeitos autónomos e responsáveis, capazes de tomar conta de si próprios. As intervenções socioeducativas têm como objetivo principal reconduzir essas ovelhas perdidas, essas almas danadas na justa direção, reforçando sua moralidade e sua autonomia através do empoderamento que irá os levar à emancipação.

O projeto assim teve como objeto as técnicas e os programas empregues para moldar, corrigir, regulamentar, e orientar o comportamento dessas populações não conformes na direção de específicos modelos de corpo, saúde, sexualidade, bem-estar e projeto de vida.

O nosso trabalho em conjunto - através da análise dos mais diversos campos etnográficos, atravessando fronteiras geográficas e disciplinares, e passando das crianças e jovens imigrantes, jovens em conflito com a lei, aos trabalhadores do sexo e aos usuários de droga - evidenciou como os programas assistencialistas destinados a reduzir o risco e a proteger os vulneráveis acabam para propor formas normativas de cidadania e subjetividade, fundadas habitualmente em assunções morais culturalmente específicas de cariz etnocêntrico. Por meio da análise da construção de específicos "problemas" e de supostas "soluções", para o atingimento de um "resultado ideal”, os trabalhos realizados pelos investigadores das duas equipas - que foram, no curso da duração projeto, apresentados e discutidos em mesas redondas e grupos de trabalhos por nós organizados - exploram como essas intervenções sociais são inerentemente discriminantes e racializadas, realçando a enorme importância na análise de marcadores de gênero, etnia e classe, entre outros.

Analisando os discursos dos profissionais da área social e de saúde, de forma crítica e engajada, colocando muitas vezes os pesquisadores em posicionamentos oblíquos e ambíguos entre diferentes competências (como antropólogos e educadores ou como profissionais da saúde), os trabalhos aqui reunidos discutem a violência institucional com que as populações alvo se deparam quando o sector social intervém nas suas vidas - normativizando a sua conduta, corporalidade, moral e escolhas -, realçando a dubiedade e contradição presentes na lógica de proteção e correção dessas políticas.

O campo de intervenção médica, em particular, revela na base das pesquisas aqui apresentadas um papel central na despolitização do governo da vida e da morte, da saúde e da doença, na tentativa de levar as pessoas a terem hábitos saudáveis, a reduzirem riscos, a terem certas concepções normalizadas do seu corpo e das suas práticas; na pedagogia, enfim, daquilo que medicamente é considerado um corpo saudável, justificando até a implementação de práticas que podem ser consideradas como não liberais, como medidas coercitivas e de internação compulsória, em nome de garantir melhorias no bem-estar coletivo. Os trabalhos desenvolvidos, em parte apresentados neste dossiê, mostram como a patologização de certas populações - em termos de classe, etnicidade, gênero, origem, estilo de vida ou religião - (re)cria fronteiras e espaços de marginalidade social e simbólica que replicam a separação histórica já muito analisada e discutida entre metrópoles e colónias, com as suas políticas de raça, pureza e higiene (Bashford, 2004; Briggs e Mantini-Briggs, 2003; Berthold, 2010). Os trabalhos mostram como a noção de higiene - mental, corporal, habitacional, sexual, parental, doméstica -, com as suas conotações de civilização, educação e moralidade, torna-se o metro para medir a capacidade de as populações marginais se tornarem cidadãs 
dignas, competentes e capazes de se cuidar (Briggs, 2003). O discurso da saúde, presente em muitos dos trabalhos aqui apresentados, possui uma dimensão normalizadora que encoraja a adopção de práticas e comportamentos prescritos, que resumem o que é ser "moderno", "ocidental", "civilizado".

Partindo de uma crescente linha de investigação preocupada com as formas de governo no liberalismo avançado, mas fazendo uma contribuição significativa com pesquisa etnográfica que tem estado ausente nesse campo, o trabalho desenvolvido nesses anos, e em parte apresentado neste dossiê, pretende analisar os múltiplos níveis em que as ideias, projetos e técnicas tentaram influenciar e transformar o comportamento das populações considerado como de risco de forma a alinhá-lo com as ideias de ordem social e bem-estar da sociedade de acolhimento, tentando gerar cidadãos competentes, capazes de se governar a si mesmos.

O interesse último da nossa pesquisa foi, portanto, olhar para a contradição aparente entre os objectivos de proteção e correção evidentes nesses programas e políticas sociais, questionando as formas de problematização - da configuração histórica de um dado objecto de intervenção aos projetos elaborados com um determinado fim em vista - e os campos de ação (instituições, atores, saberes, instrumentos), a partir dos quais se promove a gestão, a monitorização e a orientação das características físicas, mentais, comportamentais ou morais de determinadas populações. Olhando para esse tipo particular de tecnologias de cidadania no Portugal contemporâneo, prestamos particular atenção, por um lado, aos processos pelos quais certos modos de vida têm sido considerados particularmente problemáticos, sobretudo às questões do trabalho, da saúde e da pobreza e, por outro, à trajetória que liga a orientação das políticas públicas ao desenvolvimento das instituições e práticas de apoio social, à forma como as populações alvo das intervenções sociais articulam essas normas com as suas próprias estratégias de vida.

Objecto último das nossas pesquisas não foram, portanto, as políticas públicas, as instituições de solidariedade ou as pessoas em situação de pobreza, de trabalho sexual, de crime ou de dependência de drogas, como se constituíssem diferentes níveis de análise do macro ao micro, mas sim a análise da própria constituição de um campo de problematização e intervenção sobre a conduta humana, que não pode ser concebida ou praticada fora dessa mesma configuração. Pesquisar numa perspectiva de interface, trabalhando nas margens e nas fronteiras, não é, nesse sentido, estudar a graduação qualitativa da intervenção ou da experiência de uma pobreza preexistente, mas sim analisar a relação entre os diferentes elementos que estão presentes na emergência de um campo chamado pobreza - no caso, ligada à presença incomoda da vida excedente (Cohen e Shenton, 1996; Duffield, 2007) do socialmente e culturalmente outro - no qual e perante o qual tanto as instituições como a ação humana se passam a definir.

\section{Referências}

ANDERSON, M. The transformation of border controls: a European precedent? In: ANDREAS, P.; SNYDER, T. (Ed.). The wall around the west: state borders and immigration controls. Lanham: Rowman \& Littlefield, 200o. p. 15-29.

BASHFORD, A. Imperial hygiene: a critical history of colonialism, nationalism and public health. London: Palgrave, 2004.

BERTHOLD, D. Tidy whiteness: a genealogy of race, purity, and hygiene. Ethics \& the Environment, Bloomington, v. 15, n. 1, p. 1-26, 2010. BIEBRICHER, T. Faith-based initiatives and pastoral power. Economy and Society, London, v. 40, n. 3, p. 399-420, 2011.

BIGO, D. Globalized (in)security: the field and the ban-opticon. In: BIGO, D.; TSOUKALA, A. (Ed.). Terror, insecurity and liberty: illiberal practices of liberal regimes after 9/11. New York: Routledge, 2008. p. 109-156.

BIGO, D. Security, surveillance and democracy. In: BALL, K.; HAGGERTY, K.; LYON, D. The international handbook of surveillance studies. London: Routledge, 2011. p. 277-284.

BIGO, D.; GUILD, E. Policing at a distance: Schengen visa policies. In: BIGO, D.; GUILD, E. (Ed.). Controlling frontiers: free movement into and within Europe. Aldershot: Ashgate, 2005. p. 233-262. 
BRIGGS, C. Why nation-states can't teach people to be healthy: power and pragmatic miscalculation in public discourses on health. Medical Anthropology Quarterly, Malden, v. 17, n. 3, p. 287321, 2003.

BRIGGS, C.; MANTINI-BRIGGS, C. Stories in the time of cholera: racial profiling during a medical nightmare. Berkeley: University of California, 2003.

COHEN, M.; SHENTON, R. Doctrines of development. London: Routledge, 1996.

COUTIN, S. Borderlands, illegality and the spaces of non-existence. In: PERRY, R. W.; MAURER, B. (Ed.). Globalization under construction: governmentality, law, and identity. Minneapolis: University of Minnesota, 2003. p. 171-202.

DEAN, M. The constitution of poverty: toward a genealogy of liberal governance. London: Routledge, 1991.

DUFFIELD, M. Governing the borderlands: decoding the power of aid. Disasters, Malden, v. 25, n. 4, p. 308-320, 2001.

DUFFIELD, M. Development, security and unending war: governing the world of peoples. Cambridge: Polity, 2007.

EVES, R. Pentecostal dreaming and technologies of governmentality in a Melanesian society. American Ethnologist, Davis, v. 38, n. 4, p. 758-773, 2011.

EWALD, F. L'état providence. Paris: Grasset, 1986.

FASSIN, D. Policing borders, producing boundaries: the governmentality of immigration in dark times. Annual Review of Anthropology, Palo Alto, v. 40, p. 213-226, Oct. 2011.

FISCHER, N. Les corps-frontières: atteinte physique et expertise médicale dans un centre de rétention administrative. In: FASSIN, D. (Ed.). Les nouvelles frontières de la société française. Paris: Découverte, 2010. p. 477-498.

GARMANY, J. Religion and governmentality: understanding governance in urban Brazil. Geoforum, Dublin, v. 41, n. 6, p. 908-918, 2010.

GRAHAM, M. Emotional bureaucracies: emotions, civil servants, and immigrants in the Swedish welfare state. Ethos, Malden, v. 30, n. 3, p. 199-226, 2002.
GREENHALGH, S. Planned births, unplanned persons: "population" in the making of Chinese modernity. American Ethnologist, Davis, v. 3o, n. 2, p. 196-215, 2003.

HORN, D. G. Social bodies: science, reproduction, and Italian modernity. Princeton: Princeton University, 1994.

HUYSMANS, J.; DOBSON, A.; PROKHOVNIK, R. The politics of protection: sites of insecurity and political agency. London: Routledge, 2006.

INDA, J. X. Borderzones of enforcement: criminalization, workplace raids, and migrant counterconducts. In: SQUIRE, V. (Ed.). The contested politics of mobility: borderzones and irregularity. London: Routledge, 2011. p. 74-90.

KHOSRAVI, S. "Illegal" traveller: an autoethnography of borders. Hampshire: Plagrave Macmillan, 2010.

KING, N. B. Security, disease, commerce: ideologies of postcolonial global health. Social Studies of Science, Kingston, v. 32, n. 5/6, p. 763789, 2002.

LUPTON, D. Risk. London: Routledge, 2005.

O’MALLEY, P. Risk, power, and crime prevention. Economy and Society, London, v. 21, n. 3, p. 252275, 1992.

ONG, A. Making the biopolitical subject: Cambodian immigrants, refugee medicine, and cultural citizenship in California. Social Science and Medicine, Boston, v. 40, n. 9, p. 1243-1257, 1995.

PROCACCI, G. Gouverner la misère: la question sociale en France, 1789-1848. Paris: Seuil, 1993.

PUSSETTI, C.; BARROS, V. The care of the immigrant self. International Journal of Migration, Health and Social Care, Sussex, v. 8, n. 1, p. 42-51, 2012.

PUSSETTI, C.; BRAZZABENI, M. Sofrimento social: idiomas da aflição e políticas assistenciais. Etnográfica, Lisboa, v. 15, n. 3, p. 467-478, 2011.

RABINOW, P. French modern: norms and forms of the social environment. Cambridge: MIT, 1989. 
ROBERTS, D. Global governance and biopolitics: regulating human security. London: Zed Books, 2010.

ROSE, N. Inventing our selves: psychology, power, and personhood. Cambridge: Cambridge University, 1998.

RUHL, L. Liberal governance and prenatal care: risk and regulation in pregnancy. Economy and Society, London, v. 28, n. 1, p. 95-117, 1999.

SARGENT, C.; LARCHANCHÉ, S. Transnational migration and global health: the production and management of risk, illness, and access to care. Annual Review of Anthropology, Palo Alto, v. 4o, n. 1, p. 345-361, 2011.
SILVERSTEIN, P. A. Immigrant racialization and the new savage slot: race, migration and immigration in the New Europe. Annual Review of Anthropology, Palo Alto, v. 34, p. 363-384, October 2005 .

WEIR, L. Recent developments in the government of pregnancy. Economy and Society, London, v. 25, n. 3, p. 372-392, 1996. 